\title{
$V R N-1$ gene- associated prerequisites of spring growth habit in wild tetraploid wheat $T$. dicoccoides and the diploid A genome species
}

Andrey B Shcherban*, Kseniya V Strygina and Elena A Salina

\begin{abstract}
Background: In order to clarify the origin of spring growth habit in modern domesticated wheat, allelic variability of the VRN-1 gene was investigated in a wide set of accessions of the wild tetraploid species Triticum dicoccoides (BBAA), together with diploid species T. monococcum, T. boeoticum and T. urartu, presumable donors of the A genome to polyploid wheats.

Results: No significant variation was found at the VRN-B1 locus of T. dicoccoides, whereas at VRN-A1 a number of previously described alleles were found with small deletions in the promoter (VRN-A1b, VRN-A1d) or a large deletion in the first $\left(1^{\text {st }}\right)$ intron (VRN-A1L). The diploid A genome species were characterized by their own set of VRN-1 alleles including previously described VRN-A1f and VRN-ATh alleles with deletions in the promoter region and the VRN-A1ins allele containing a $0.5 \mathrm{~kb}$ insertion in the $1^{\text {st }}$ intron. Based on the CAPS screening data, alleles VRN-Alf and VRN-Alins were species-specific for T. monococcum, while allele VRN-ATh was specific for T. boeoticum. Different indels were revealed in both the promoter and $1^{\text {st }}$ intron of the recessive VRN-ATU allele providing specific identification of T. urartu, the proposed donor of the A genome to modern wheat. We found that alleles VRN-A1b and VRN-A1h, previously described as dominant, have either no or weak association with spring growth habit, while in some diploid accessions this habit was associated with the recessive VRN-A1 allele.
\end{abstract}

Conclusions: Spring growth habit in diploid wheats was only partially associated with indels in regulatory regions of the VRN-1 gene. An exception is T. monococcum where dominant mutations in both the promoter region and, especially, the $1^{\text {st }}$ intron were selected during domestication resulting in a greater variety of spring forms. The wild tetraploid T. dicoccoides had a distinct set of VRN-A1 alleles compared to the diploids in this study, indicating an independent origin of spring tetraploid forms that likely occurred after combining of diploid genomes. These alleles were subsequently inherited by cultivated polyploid (tetraploid and hexaploid) descendants.

Keywords: Allelic diversity, Vernalization, VRN-1 gene, Promoter, First intron, Tetraploid, Diploid, Triticum

\section{Background}

Triticum dicoccoides or T. turgidum ssp. dicoccoides Thell is considered to be the wild form of all domesticated emmer wheats [1]. The species has two homologous sets of chromosomes (BBAA) thought to have arisen from spontaneous hybridization between two diploid donor species. The donor of the $\mathrm{B}$ genome is unknown but was likely a species closely related to modern Aegilops speltoides Tausch $(2 \mathrm{n}=14$; SS) [2-4]. Potential sources of the A genome are represented by three closely related species:

\footnotetext{
*Correspondence: atos@bionet.nsc.ru

Institute of Cytology and Genetics, Lavrentiev ave. 10, Novosibirsk 630090, Russia
}

T. urartu Tum. ex Gandil., T. boeoticum Boiss. and T. monococcum L. These species are separated by crossing barriers [5], and differ in their plant morphology [6] and biochemical and molecular marker loci $[3,7,8]$. $T$. boeoticum is considered to be the progenitor of cultivated diploid einkorn wheat, T. monococcum. T. urartu is considered as the donor of A genome to the wild tetraploid species: $T$. dicoccoides (BBAA) and $T$. araraticum (GGAA) [9], which was subsequently inherited by other important polyploid wheat species including $T$. durum (BBAA), T. timopheevii (GGAA), and common wheat $T$. aestivum (BBAADD). Despite many studies of Triticum 
domestication, employing morphological, cytological $[6,10]$ and molecular marker analysis techniques [7,11-14], many questions remain unanswered with respect to the phylogenetic relationships between diploid wheat species and between diploids and their polyploid descendants.

Adaptability of polyploid wheat to a wide range of environments has been at least partially facilitated by allelic diversity in $V R N$ genes regulating growth habit and flowering time [15]. Wheats are categorized into two major forms: those requiring vernalization or exposure to cold to accelerate flowering (winter forms) and those which do not require vernalization (spring forms). Vernalization prevents floral initiation during winter, thereby protecting sensitive floral meristems from freezing temperatures. In recent years, researchers have significantly advanced our understanding of the molecular mechanisms of vernalization in wheat. The genomic locations and sequences of three vernalization genes (VRN-1,VRN-2 and $V R N-3$ ) responsible for spring/winter growth habit have been determined [16-18].

The $V R N-1$ gene is proposed as the main initiating factor of the regulatory cascade initiating flowering $[16,19]$. VRN-1 encodes a MADS-box transcription factor which controls the transition of the vegetative shoot apical meristem to the reproductive phase [20,21]. In vernalization-requiring cereals, $V R N-1$ expression is induced by vernalization, with the level of expression being dependent on the length of cold exposure [22]. VRN-1 downregulates the floral repressor $V R N-2$, and allows long-day induction of the floral activator $V R N-3$ to accelerate subsequent stages of floral development $[17,19]$.

Changes in the growth habit of winter wheat to spring wheat are primarily due to dominant mutations in regulatory regions (promoter or intron 1) of $V R N-1$ [15]. VRN$A 1$ alleles of the hexaploid or common wheat $T$. aestivum L. $(2 \mathrm{n}=42, \mathrm{BBAADD})$ and the tetraploid T. turgidum $\mathrm{L}$. $(2 \mathrm{n}=28, \mathrm{BBAA})$, have so far been identified containing nucleotide deletions as well as insertions of mobile elements in both regions [23,24]. By contrast, dominant mutations in VRN-B1 and VRN-D1 genes are predominantly caused by large deletions in the $1^{\text {st }}$ intron [24]. Recently, a novel $V R N-B 1$ allele was identified as having a deletion coupled with sequence duplication within intron 1 [25-27].

Most of the wild Triticeae species have a winter growth habit, suggesting that the recessive $V R N-1$ allele is the ancestral form. By contrast, there are many cultivated polyploid wheats with a spring growth habit and with at least one dominant $V R N-1$ allele [28]. Reports demonstrate that different combinations of dominant and recessive $V R N-1$ alleles (VRN-1 genotype) significantly affect the time of flowering $[29,30]$. Spring growth habit determined by dominant $V R N-1$ alleles could be either inherited from ancestral diploids or could result from selection of independent mutations appearing during the adaptation to different environments after domestication. To resolve this issue, a large scale screening of $V R N-1$ polymorphisms among both diploid and polyploid wheat species is required. To date, a limited number of accessions of diploid and tetraploid progenitors of T. aestivum have been examined $[16,23,24,31,32]$.

In the present study, molecular variability of $V R N-1$ genes was analyzed based on a representative set of accessions of wild tetraploid wheat and diploid A genome species. The main objective was to identify and characterize different alleles of $V R N-1$ genes and to analyze their distribution among accessions. We also examined the influence of different alleles and their combinations on vernalization requirement. This analysis sheds light on the evolution of $V R N-1$ genes in diploids and during the first stages of wheat polyploidization. It contributes to our understanding of the phylogenetic relationships between diploid and polyploid wheat species and the evolutionary history of modern domesticated wheat.

\section{Results \\ VRN-1 allelic variability in wild tetraploid wheat \\ T. dicoccoides \\ Promoter region}

Vernalization sensitivity in tetraploid wheat $T$. dicoccoides is controlled by alleles at the 2 homoeologous loci, VRN-A1 and VRN-B1. The specific primers Vrn1AF and Int1R were used in order to identify variation in the promoter region of VRN-A1 locus as described by Yan et al. [23] (Table 1). Almost all of the 80 studied accessions of $T$. dicoccoides yielded a PCR product of approximately $0.7 \mathrm{~kb}$ (Figure 1a). To further analyse these PCR products, we digested them with a frequently-cutting restriction endonuclease $M s p$ I. The restriction patterns could be divided into two types (Figure 1b). The first type, characteristic of the vast majority of the studied accessions, contained two major fragments of $\sim 140$ and $200 \mathrm{bp}$ long. The second type was found in only 3 of the $80 \mathrm{~T}$. dicoccoides accessions and was characterized by a fragment of approximately $120 \mathrm{bp}$ in place of the $140 \mathrm{bp}$ band. To further analyze allelic variation at the VRN-A1 promoter, we selected a set of accessions representing both restriction patterns and sequenced the PCR products obtained with VrnA1F/Int1R primers (Table 2).

The VRN-A1 promoter sequences of accessions IG 113301, IG 139189, IG 46297, k-5198, belonging to the first restriction pattern type (Figure 1b) were completely identical to the known sequence of the recessive $V R N$ A1 allele from $T$. dicoccoides (AY747598) and contained an Msp I restriction fragment of $138 \mathrm{bp}$. VRN-A1 promoter sequences of all polyploid wheats both studied here and from databases contained the $8 \mathrm{bp}$ insertion and an additional $M s p$ I site characteristic of the respective 
Table 1 PCR markers for determining the presence of different alleles of VRN-A1, VRN-B1 in diploid and polyploid wheats

\begin{tabular}{|c|c|c|c|c|c|c|}
\hline PCR marker & Name & Primer $\left(5^{\prime} \rightarrow 3^{\prime}\right)$ & Target allele(s) & $\begin{array}{l}\text { Expected product } \\
\text { size (bp) }\end{array}$ & $\begin{array}{l}\text { Annealing } \\
\text { temp. }\left({ }^{\circ} \mathrm{C}\right)\end{array}$ & Reference. \\
\hline \multirow[t]{8}{*}{ VRN-A1 marker* } & Vrn1AF & GAAAGGAAAAATTCTGCTCG & VRN-Ala & 876 and 965 & 55.0 & {$[16,23,31]$} \\
\hline & Int1R & GCAGGAAATCGAAATCGAAG & VRN-A1b & 694 & & \\
\hline & & & VRN-A1d & 662 & & \\
\hline & & & VRN-A1h & 684 & & \\
\hline & & & VRN-A1f & 703 & & \\
\hline & & & $V R N-A 1$ & 704 & & \\
\hline & & & VRN-A1U & 705 & & \\
\hline & & & VRN-A1U & 713 & & \\
\hline \multirow[t]{2}{*}{ T. monococcum VRN-A1 } & Indel(-)F & CGCTCTTATATTTGTTTACCAGGG & VRN-A1 & 1025 & 50.0 & - \\
\hline & Indel(-)R & GGGTCAACTATTCTGTGGAG & VRN-A1U, VRN-A1U' & no product & & \\
\hline \multirow[t]{2}{*}{ VRN-A1 deletion of $1.4 \mathrm{~kb}$} & $\operatorname{lntr} 1 / C / F$ & GCACTCCTAACCCACTAACC & VRN-A1U, VRN-A1U' & 1068 & 56.0 & [24] \\
\hline & Intr1/AB/R & TCATCCATCATCAAGGCAAA & & & & \\
\hline \multirow[t]{2}{*}{ VRN-A1 insertion of $0.5 \mathrm{~kb}$} & Intr 1 & ATCATCTTCTCCACCAAGGG & VRN-A1ins & 1980 & 50.0 & - \\
\hline & Intr1insR & AATGAACAGCACGGAAACAG & $V R N-A 1$ & 1476 & & \\
\hline \multirow[t]{2}{*}{ VRN-A1 deletion of $7,2 \mathrm{~kb}$} & $\mathrm{Ex} 1 / \mathrm{C} / \mathrm{F}$ & GTTCTCCACCGAGTCATGGT & $V R N-A 1 L$ & 522 & 55.6 & [24] \\
\hline & Intr1/A/R3 & AAGTAAGACAACACGAATGTGAGA & & & & \\
\hline \multirow[t]{2}{*}{ VRN-B1 marker* } & P2 & TCATGCACGCACACACGGTA & $V R N-B 1$ & 814 & 55.0 & {$[25]$} \\
\hline & P5 & GGCCAACCCTACACCCCAAG & & & & \\
\hline \multirow[t]{2}{*}{ VRN-B1 Non-deletion } & Intr1/B/F & CAAGTGGAACGGTTAGGACA & $V R N-B 1$ & 1149 & 56.4 & [24] \\
\hline & Intr1/B/R4 & CAAATGAAAAGGAATGAGAGCA & & & & \\
\hline
\end{tabular}

*These diagnostic markers detect allelic variation at the promoter regions. In other cases variation within intron 1 of corresponding genes is detected.

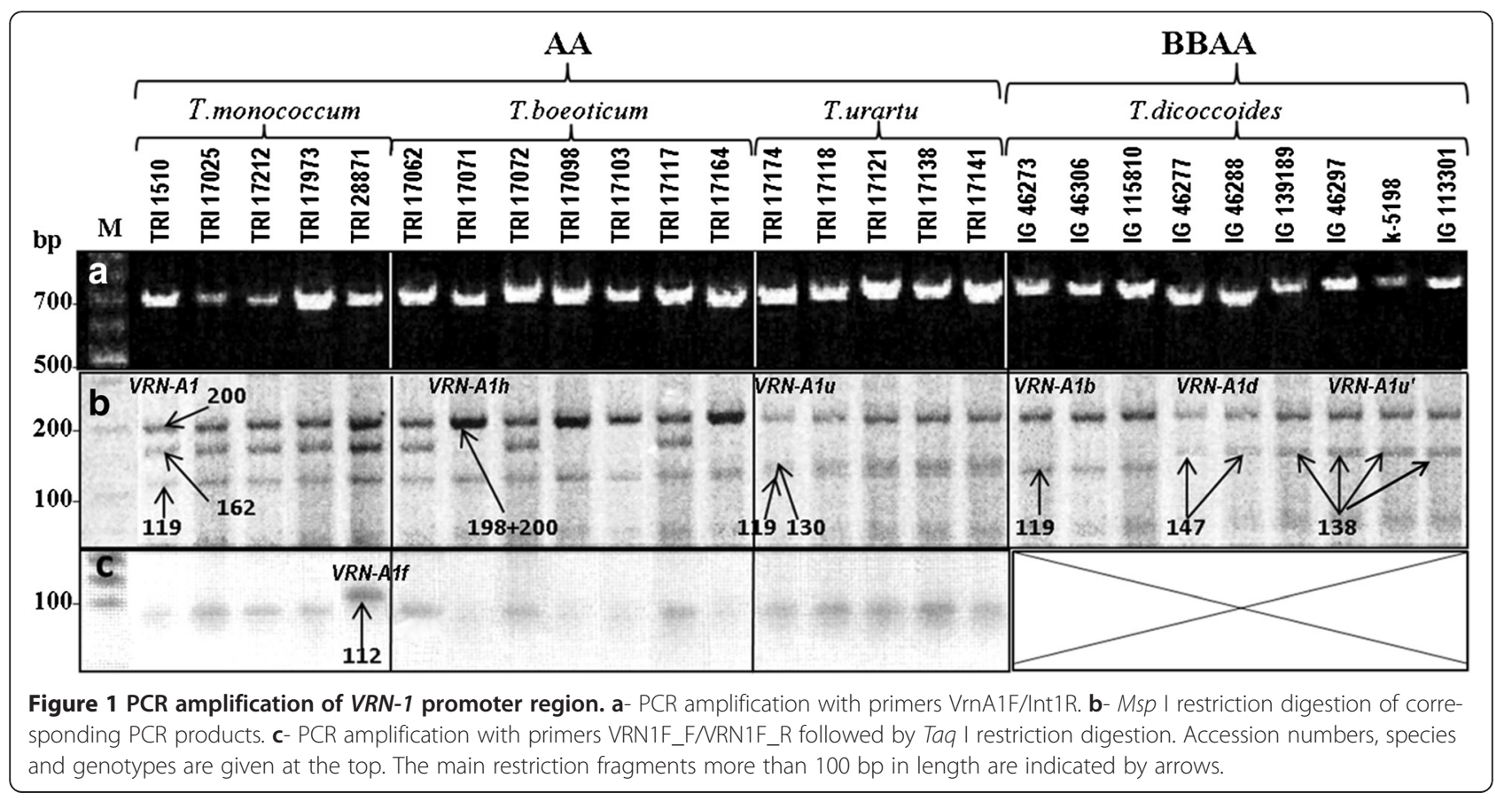


Table 2 Allelic variants of VRN-A1 gene in a selected set of accessions of diploid Triticum species and tetraploid $T$. dicoccoides

\begin{tabular}{|c|c|c|c|c|c|c|c|c|}
\hline \multirow[t]{4}{*}{ Species (genome) } & \multirow{4}{*}{$\begin{array}{l}\text { Accession } \\
\text { number }\end{array}$} & \multirow{4}{*}{$\begin{array}{l}\text { Growth type } \\
\text { (Winter/ Spring) }\end{array}$} & \multicolumn{3}{|c|}{ VRN-A1 structural forms* } & \multirow[t]{4}{*}{ Alleles** } & \multirow{4}{*}{$\begin{array}{l}\text { GenBank } \\
\text { Ac.N. }\end{array}$} & \multirow{4}{*}{$\begin{array}{l}\text { Homologous } \\
\text { sequences } \\
\text { from GenBank }\end{array}$} \\
\hline & & & \multirow[t]{3}{*}{ Promoter } & \multicolumn{2}{|l|}{ Intron 1} & & & \\
\hline & & & & Insertion & Deletions & & & \\
\hline & & & & $0.5 \mathrm{~kb}$ & $1.4 \mathrm{~kb} / 7.2 \mathrm{~kb}$ & & & \\
\hline \multirow[t]{5}{*}{ T. monococcum (AA) } & TRI 1510 & $\mathrm{~S}$ & $V R N-A 1$ & + & $-1-$ & VRN-Alins & KM586653 & AY188331 \\
\hline & TRI 17025 & S & $V R N-A 1$ & - & $-/-$ & vrn-Alvrn2 & $-/ /-$ & $-/ /-$ \\
\hline & TRI 17212 & S & $V R N-A 1$ & + & $-/-$ & VRN-Alins & $-/ /-$ & $-/ /-$ \\
\hline & TRI 17973 & $S$ & $V R N-A 1$ & - & $-1-$ & vrn-ATVRN-2 & $-/ /-$ & $-/ /-$ \\
\hline & TRI 28871 & $S$ & VRN-A1f & + & $-1-$ & VRN-A1f VRN-A1ins & KM586656 & DQ146421 \\
\hline \multirow[t]{7}{*}{ T. boeoticum (AA) } & TRI 17062 & W & VRN-A1 & - & $-1-$ & $v r n-A 1$ & KM586653 & AY188331 \\
\hline & TRI 17071 & S & VRN-A1h & - & $-/-$ & VRN-A1h & KM586657 & GQ451745 \\
\hline & TRI 17072 & $S$ & VRN-A1 & - & $-/-$ & vrn-ATVRN-2 & KM586653 & AY188331 \\
\hline & TRI 17098 & S & VRN-A1h & - & $-/-$ & VRN-A1h & KM586657 & GQ451745 \\
\hline & TRI 17103 & W & VRN-Aih & - & $-/-$ & VRN-A1h & $-/ /-$ & $-/ /-$ \\
\hline & TRI 17117 & W & $V R N-A 1$ & - & $-1-$ & $v r n-A 1$ & KM586653 & AY188331 \\
\hline & TRI 17164 & W & VRN-A1h & - & $-1-$ & VRN-A1h & KM586657 & GQ451745 \\
\hline \multirow[t]{5}{*}{ T. urartu (AA) } & TRI 17174 & W & VRN-A1U & - & $+/-$ & vrn-Alu & KM586659 & GQ482970 \\
\hline & TRI 17118 & W & VRN-A1U & - & $+/-$ & vrn-Alu & $-/ /-$ & $-/ /-$ \\
\hline & TRI 17121 & W & VRN-A1U & - & $+/-$ & vrn-A1u & $-/ /-$ & $-/ /-$ \\
\hline & TRI 17138 & W & VRN-A1U & - & $+/-$ & vrn-A1u & $-/ /-$ & $-/ /-$ \\
\hline & TRI 17141 & W & VRN-A1U & - & $+/-$ & vrn-A1u & $-/ /-$ & $-/ /-$ \\
\hline \multirow[t]{9}{*}{ T. dicoccoides (BBAA) } & IG 113301 & W & $V R N-A 1 U^{\prime}$ & - & $+/-$ & vrn-Alu' & KM586660 & AY747598 \\
\hline & IG 46273 & W & VRN-A1b & - & $+/-$ & VRN-A1b & KM586654 & AY616461 \\
\hline & IG 46306 & W & VRN-A1b & - & $+/-$ & $V R N-A 1 b$ & $-/ /-$ & $-/ /-$ \\
\hline & IG 115810 & W & VRN-A1b & - & $+/-$ & VRN-A1b & $-/ /-$ & $-/ /-$ \\
\hline & IG 46277 & $S$ & VRN-A1d & - & $+/-$ & VRN-A1d & KM586655 & AY616462 \\
\hline & IG 46288 & S & VRN-Ald & - & $+/-$ & VRN-A1d & $-/ /-$ & $-/ /-$ \\
\hline & IG 139189 & S & $V R N-A 1 u^{\prime}$ & - & $? /+$ & VRN-A1L & KM586658 & AY747598 \\
\hline & IG 46297 & S & $V R N-A 1 u^{\prime}$ & - & $? /+$ & VRN-A1L & $-/ /-$ & $-/ /-$ \\
\hline & $k-5198$ & S & $V R N-A 1 U^{\prime}$ & - & $? /+$ & VRN-A1L & $-/ /-$ & $-/ /-$ \\
\hline
\end{tabular}

* In all cases the promoter region was sequenced; insertion and deletions in intron 1 were determined by PCR (see Table 1).

${ }^{*} v r n-A 1, v r n-A 1 u, v r n-A 1 u^{\prime}$ - recessive alleles (initial structural forms); VRN-A1- structurally modified (mutated) forms.

VRN-A1u allele of T. urartu (see below). Taking into account these features, we designated the promoter sequence of the recessive $V R N-A 1$ allele of $T$. dicoccoides as $V R N-A 1 u^{\prime}$ (Figures 2 and 3; Table 2; Additional file 1).

Accessions IG 46273, IG 46306 and IG 115810 of the second type were identified to be a previously described $V R N-A 1 b$ allele [23], with a 19 bp deletion in the promoter region as compared to the recessive allele (Figure 2). This deletion created the diagnostic Msp I restriction fragment of 119 bp (Figure 3).

Two out of $80 \mathrm{~T}$. dicoccoides accessions (IG46277 and IG 46288) yielded a slightly smaller PCR product compared with the other accessions (Figure 1a). They were almost undistinguishable from the accessions of the first type by Msp I restriction (Figure 1b). Sequencing showed that these accessions contained a previously described VRN-A1d allele [23]. Compared with the VRN$A 1 b$ allele, this allele has an additional $32 \mathrm{bp}$ deletion, generating a diagnostic 147 bp Msp I restriction fragment (Figures 2 and 3).

For analysis of the $V R N-B 1$ locus the primers P2/P5 were used to amplify an approximately $0.8 \mathrm{~kb}$ region of the promoter sequence (Table 1). No differences were revealed by PCR or subsequent $M s p$ I digestion (see Additional file 2). Based on the known $V R N-B 1$ sequences of T. aestivum (AY747602-04, AY616453, AY616456), Msp I restriction fragments of 494, 104 and 128 bp were expected. We sequenced PCR products of $0.8 \mathrm{~kb}$ from 5 randomly selected accessions with diverse origins (see Additional file 1). All five sequences were highly similar to 


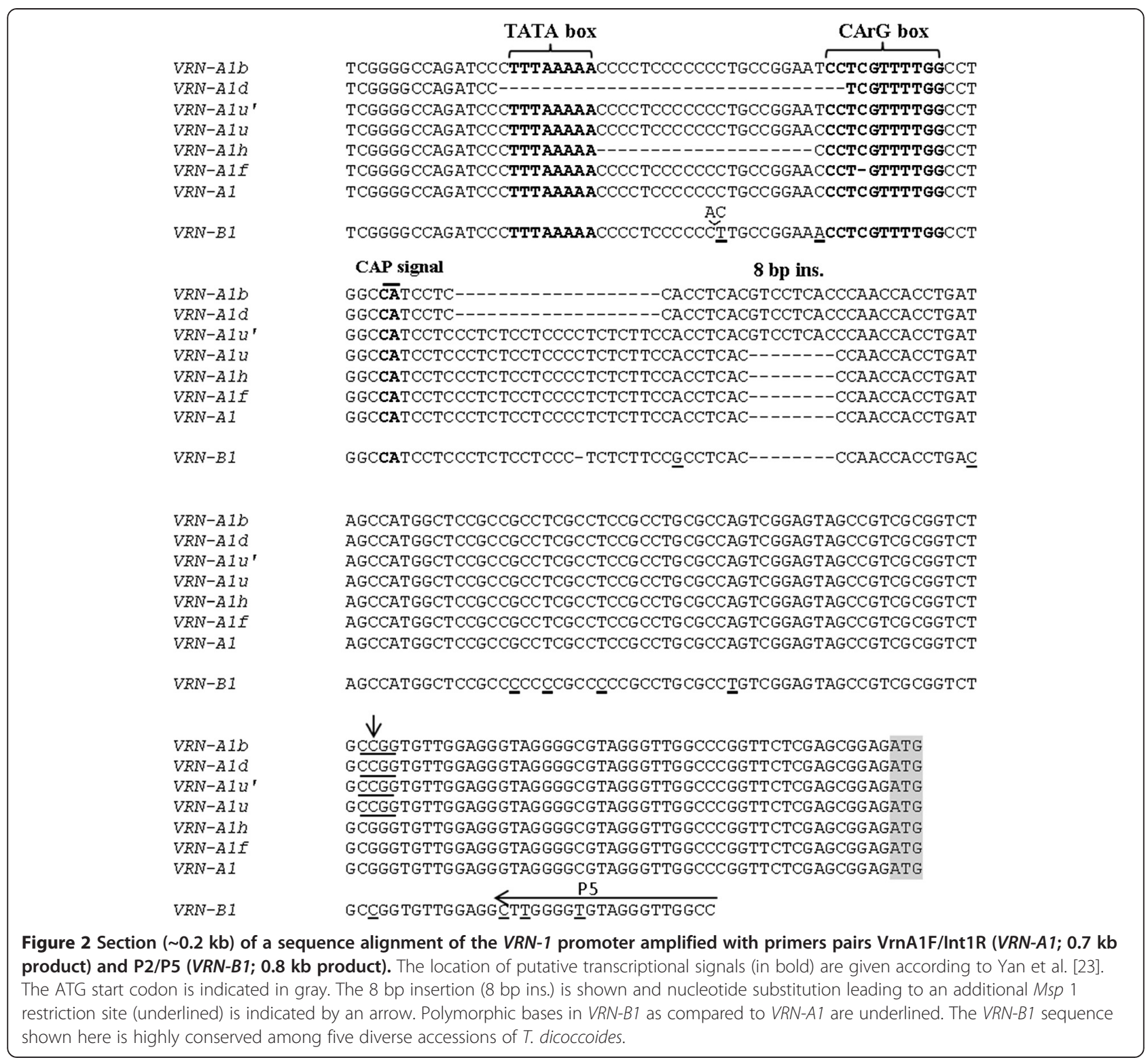

each other and to known VRN-B1 sequences (98-100\% homology). Minor variation between individual sequences was found including small deletions of up to $7 \mathrm{bp}$, however, almost all of this variation was upstream from the conserved region containing putative transcriptional signals (Figure 2). The VRN-B1 sequences yielded in this study were deposited to Genbank under Ac.N. KM586661-65.

\section{$1^{\text {st }}$ intron}

The primer pair Intr $1 / \mathrm{C} / \mathrm{F}$ and $\operatorname{Intr} 1 / \mathrm{AB} / \mathrm{R}$ for the $1^{\text {st }}$ intron of $V R N-A 1$ (Table 1; Figure 4) were previously used to confirm the presence of the recessive allele of T. aestivum [24]. This allele contains a $1.4 \mathrm{~kb}$ deletion compared with the respective alleles of $T$. monococcum and $T$. boeoticum (see below). Almost all the studied accessions of $T$. dicoccoides yielded a PCR product of approximately $1 \mathrm{~kb}$, indicating the presence of the deletion, except for accessions k-5198, IG 139189 and IG 46297 which gave no products (Figure 5c). Previously, $V R N-A 1$ allele from the tetraploid variety 'Langdon' (AY747598) was determined as having a $7.2 \mathrm{~kb}$ deletion in intron 1. Using the primers pair Ex1/C/F and Intr1/A/R3 for detection of this allele (Table 1; Figure 4), the three $T$. dicoccoides accessions generated a PCR product of about $0.5 \mathrm{~kb}$ (Figure $5 \mathrm{~d}$ ) identical to the corresponding sequence from 'Langdon'. Here, we refer to this allele as $V R N-A 1 L$ (Figure 4; Table 2). 


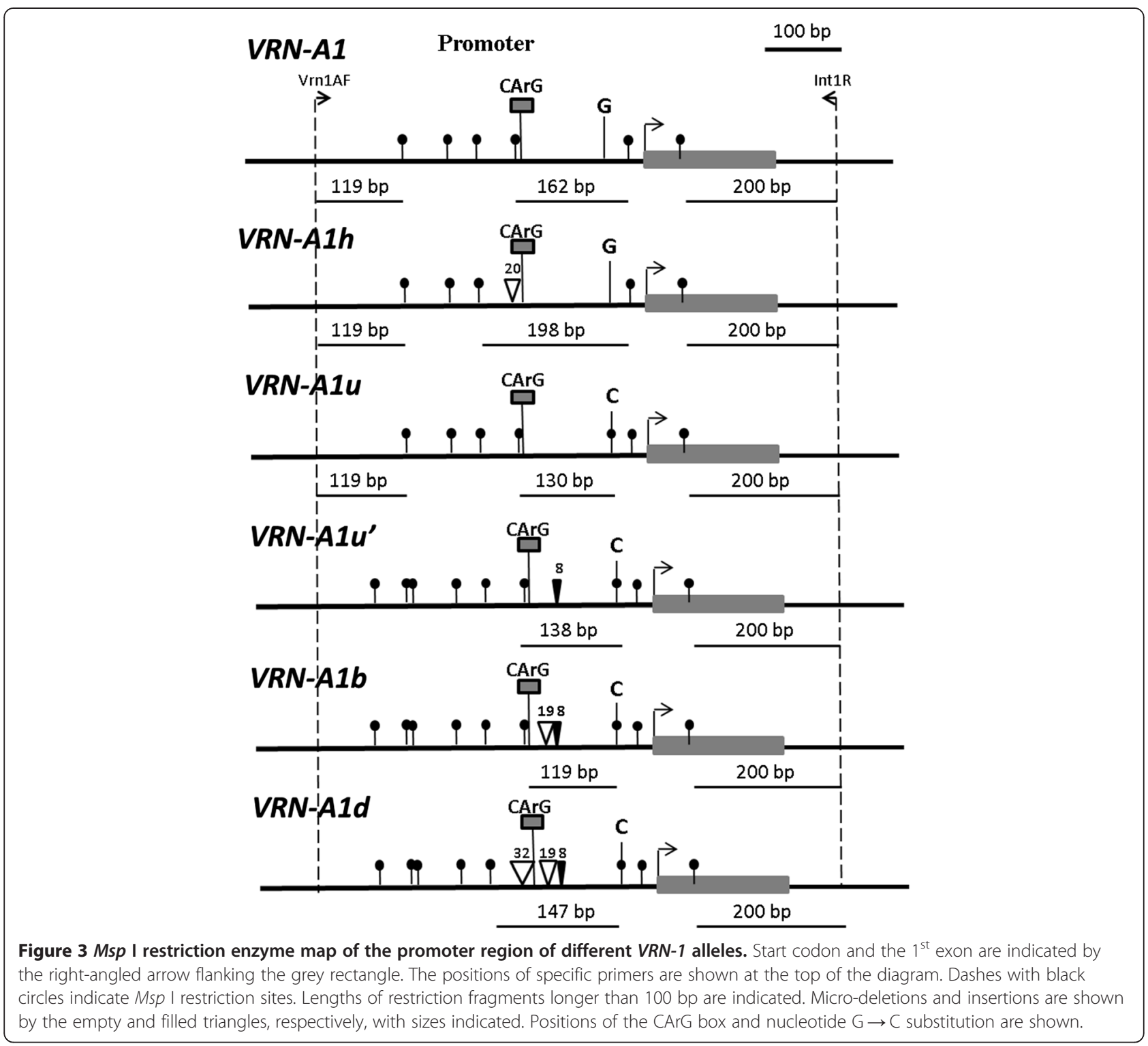

The pair of primers $\operatorname{Intr} 1 / \mathrm{B} / \mathrm{F}$ and $\operatorname{Intr} 1 / \mathrm{B} / \mathrm{R} 4$ was designed as a positive control for the absence of large mutations (deletions or insertions) in intron 1 of the VRN-B1 locus [24] (Table 1). Using these primers, all accessions of $T$. dicoccoides yielded a $1149 \mathrm{bp}$ PCR product indicating the presence of the wild recessive $V R N-B 1$ allele (see Additional file 2).

Thus, tetraploid $T$. dicoccoides displayed structural variation both at the promoter and $1^{\text {st }}$ intron regions of $V R N-A 1$ locus, whereas at VRN-B1 no significant differences were found in the studied accessions. To determine whether this variation originated at the tetraploid level or in diploid donors of the A genome, we conducted an analysis of diploid progenitor species.
VRN-1 allelic variability in diploid Triticum species

\section{Promoter region}

We analysed VRN-1 promoter variability in 160 accessions of the diploid wheat species $T$. monococcum, $T$. boeoticum and T. urartu (Additional file 1). As for the tetraploid accessions, the diploids yielded PCR products of $\sim 0.7 \mathrm{~kb}$ with primers Vrn1AF and Int1R (Figure 1a). Msp I- digestion yielded three patterns of restriction (Figure 1b). The first pattern contained bands of 119, 162 and $200 \mathrm{bp}$, consistent with the known recessive VRN-A1 allele of T. monococcum (AY188331). This pattern was characteristic of all 61 accessions of T. monococcum and 39 accessions of T. boeoticum (Additional file 1). The remaining 20 accessions of $T$. boeoticum yielded a 

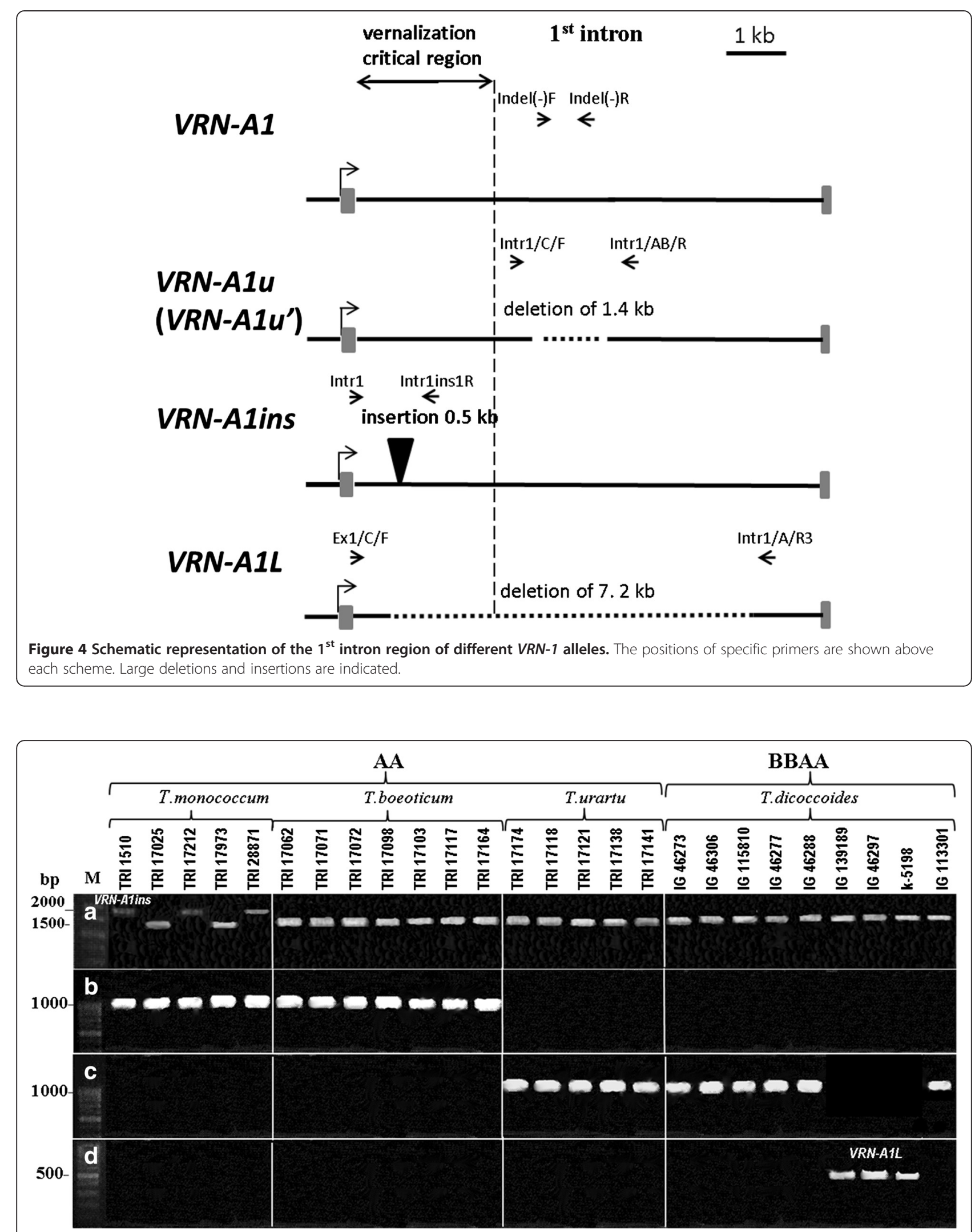

Figure 5 PCR amplification of VRN-1 intron 1 region. PCR amplification with primers Intr1/Intr1 insR (a), Indel(-)F/Indel(-)R (b), Intr1/C/F//Intr1/ $A B / R$ (c), Ex1/C/F//Intr1/A/R3 (d). Accession numbers, species and genotypes are given at the top. 
pattern with no middle band at $162 \mathrm{bp}$, and intensive 200 bp band. All accessions of T. urartu were distinguished from the other two species by a double band of about 120-130 bp (Figure 1b). To further analyze allelic variation in the $V R N-A 1$ promoter, we selected a subset of accessions representing the three restriction patterns and sequenced the corresponding PCR products obtained with VrnA1F/Int1R primers (Table 2).

Among five selected accessions of $T$. monococcum, four had identical promoter $V R N-A 1$ sequences to the reported $V R N-A 1$ allele (AY188331) (Table 2). Accession TRI 28871 had a 1 bp deletion in a CArG-box motif in the promoter region (Figure 2). This allele has been previously identified in a spring accession of $T$. monococcum as VRN-A1f [16]. CAPS marker screening of all diploid accessions (see Methods) revealed a 112 bp band characteristic of $V R N-A 1 f$ (Figure 1c) in three more accessions of $T$. monococcum (Additional file 1). Subsequent sequencing confirmed the presence of $V R N-A 1 f$ in these accessions.

Of seven selected accessions of $T$. boeoticum, three had promoter sequences which were identical to the recessive VRN-A1 allele of T. monococcum (Table 2). Sequences of the remaining four accessions were identical to the previously described $V R N-A 1 h$ allele (GQ451745), which differs from $V R N-A 1$ by a 20 bp deletion near the CArG- box (Figure 2). This deletion resulted in a loss of one Msp I site (Figure 3), explaining the absence of the $160 \mathrm{bp}$ band and increased staining intensity of the $200 \mathrm{bp}$ band due to superposition of a $198 \mathrm{bp}$ fragment (Figure 1b). We concluded that the $20 \mathrm{~T}$. boeoticum accessions showing this restriction pattern carry VRN$A 1 h$, whereas the remaining accessions are likely to have $V R N-A 1$ (Additional file 1).

The five selected accessions of $T$. urartu had an identical $V R N-A 1$ promoter sequence (Table 2), with $98 \%$ sequence identity to the respective sequence of $T$. monococcum VRN-A1. Of the few nucleotide substitutions, one ( $\mathrm{G} \rightarrow \mathrm{C}$, position -48 from the start codon) resulted in a new Msp I restriction site (Figure 2, 3). This explains the appearance of a $130 \mathrm{bp}$ band coupled with the loss of the $160 \mathrm{bp}$ band (Figure 1b). Based on our restriction analysis across the three diploid species, this substitution is specific for $T$. urartu, so we have designated the corresponding allele as $V R N-A 1 u$ (Additional file 1).

\section{$1^{\text {st }}$ intron}

Alignment of the known $V R N-A 1$ sequences from databases at the $1^{\text {st }}$ intron revealed two large indels (Figure 4). An insertion of $0.5 \mathrm{~kb}$ (VRN-A1ins) was found previously in two spring accessions of $T$. monococcum near the $5^{\prime}$ side of intron 1 [31]. A deletion of $1.4 \mathrm{~kb}$ located about $4 \mathrm{~kb}$ downstream from the start of intron 1 was found in all known $V R N-A 1$ sequences obtained from polyploid wheat species in comparison with T. monococcum.

We designed specific primers flanking both indels to study variation at the $1^{\text {st }}$ intron of $V R N-A 1$ in three diploid species (Table 1; Figure 4). The primers Indel(-)F and Indel(-)R allow identification of the $V R N-A 1$ allele of $T$. monococcum without the deletion of $1.4 \mathrm{~kb}$, with an expected PCR product of approximately $1 \mathrm{~kb}$. All accessions of T. monococcum and T. boeoticum yielded this product, whereas no product was obtained with the $T$. urartu accessions (Figure 5b). The primers Intr1/C/F and Intr1/AB/R flanking the $1.4 \mathrm{~kb}$ deletion were used as a positive amplification control for the presence of this deletion in T. urartu (Table 1; Figure 4). As for $T$. dicoccoides (see above), all T. urartu accessions yielded the $1 \mathrm{~kb}$ product. This product was absent for all accessions of T. monococcum and T. boeoticum, confirming the previous result (Figure $5 \mathrm{c}$ ). Hence, the $1.4 \mathrm{~kb}$ deletion in intron 1 is a specific marker for the $V R N-A 1 u$ (VRN-A1 $\left.u^{\prime}\right)$ allele of $T$. urartu and polyploid species with an A-genome (Additional file 1).

Using primer pair Intr1/Intr1insR, 19 accessions of $T$. monococcum generated a $2 \mathrm{~kb}$ PCR product, demonstrating that these accessions carried VRN-A1ins (accessions TRI 1510, 17212 in Figure 5a; additional file 1). The remaining $42 T$. monococcum accession yielded a fragment of about $1.5 \mathrm{~kb}$ indicating the absence of $V R N$ Alins. Similarly, no VRN-A1ins was found in any of the T. urartu or T. boeoticum accessions, excepting a single $T$. boeoticum accession from Europe (which may have been mis-classified) (Additional file 1).

\section{Evaluation of growth habit in diploid and tetraploid accessions}

To assess the impact of $V R N-1$ alleles on vernalization requirements in diploid and tetraploid wheats, we selected 26 accessions containing both initial (recessive), or structurally modified forms of $V R N-1$ genes (Table 2). Noteworthy, in two accessions of T. monococcum and 1 accession of $T$. boeoticum the recessive form of $V R N-A 1$ (VRN-A1 promoter, no insertion in intron 1) was associated with spring growth habit, implying an involvement of other genes in the determination of this habit. Four accessions of T. monococcum carrying a VRN-A1f allele was defined as a spring wheat. Out of four accessions of $T$. boeoticum carrying the VRN-A1h allele, two had a spring growth habit, while the other two were winter types. All five accessions of $T$. urartu with uniform $V R N-A 1$ sequences were winter types (Table 2 ).

With respect to the tetraploid $T$. dicoccoides, allele $V R N-A 1 b$ was associated with winter growth habit in all three accessions containing this allele, whereas VRN$A 1 d$ and $V R N-A 1 L$ were strongly associated with spring habit (Table 2). Accession IG 113301 with a recessive 
form of the $V R N-A 1$ gene $\left(V R N-A 1 u^{\prime}\right.$ promoter, no changes in intron 1) was classified as a winter wheat.

\section{Discussion}

Previous studies of $V R N-1$ allelic variability in wild Triticum species were restricted either by inclusion of a limited number of accessions for analysis $[16,23,24,31]$, or by the use of a single regulatory region ( $V R N-1$ promoter) as a marker [32]. Here we have made an attempt to systematically study these species using a wide set of accessions representing wild tetraploid $T$. dicoccoides and three diploid A genome progenitor species (Additional file 1).

\section{VRN-1 allelic diversity in diploid A genome species}

A CAPS markers designed to the promoter $V R N-1$ region allowed us to reveal the three most frequent allelic variants in diploids, while avoiding a large scale sequencing approach (Figure 1). The recessive VRN-A1 allele was prevalent among the accessions of $T$. monococcum and T. boeoticum (Additional file 1; Table 2). Twenty accessions of $T$. boeoticum contained allele VRN-A1h which was absent from all accessions of $T$. monococcum. We found the previously described VRN-A1f allele (Figure 2) in four accessions of T. monococcum (Table 2; Additional file 1).

All accessions of $T$. urartu had a specific restriction pattern with the frequent base cutting enzyme, Msp I, allowing us to distinguish $T$. urartu from the other diploid species (Figure 1b). Sequencing determined that the corresponding $V R N-A 1 u$ allele is highly homologous to the known VRN-A1 alleles of polyploid wheat species. The majority of these alleles contain an 8 bp insertion which is absent in the respective sequences of diploids (Figures 2 and 3), except for a single previously studied sequence from $T$. urartu (GQ451737). We have classified the latter allele as $V R N-A 1 u^{\prime}$, suggesting that it originated from the basic VRN-A1u allele of T. urartu and was subsequently inherited by polyploid descendants bearing an A-genome, including T. dicoccoides (Additional file 1).

Previously, molecular markers were designed for identification of large deletions in intron 1 affecting vernalization response [24]. Using these and our own markers (Table 1) we found two major alterations occurring at different evolutionary stages of diploid wheats (Figures 4 and 5). The insertion of $0.5 \mathrm{~kb}$ may have occurred relatively recently during the evolution of $T$. monococcum, since it was found only in some accessions of this species (Additional file 1). The deletion of $1.4 \mathrm{~kb}$ probably occurred in a progenitor of $T$. urartu after its divergence from $T$. boeoticum. The estimated divergence time between T. boeoticum and T. urartu and between T. monococcum and T. boeoticum is 570000 and 290000 YA, respectively [33]. Besides T. dicoccoides, the $1.4 \mathrm{~kb}$ deletion is characteristic of other polyploid species with an A genome.
Thus, the analysis of both the promoter and $1^{\text {st }}$ intron regions of $V R N-1$ gene in diploid wheat species allowed us to reveal species-specific alleles including $V R N-A 1 f$ and VRN-Alins for T. monococcum, VRN-A1h for T. boeoticum and VRN-A1u for T. urartu. The structural similarity of the latter allele with the respective alleles of wheat polyploids confirmed T. urartu as the donor of the A genome to wheat polyploids.

\section{Tetraploid wheat genetic diversity at VRN-1 loci; the beginning of the formation of a new allelic set}

Wild emmer wheat $T$. dicoccoides as a species is about 360,000 years old, resulting from a spontaneous hybridization event which took place somewhere in the Fertile Crescent, a crescent-shaped area of fertile land in the Middle East [34]. T. dicoccoides belongs to the first cereals domesticated by humans and it is this domestication step which provided the key for subsequent bread wheat evolution [1].

Using the same approach as for the diploid wheats to study $T$. dicoccoides, we found two previously described alleles $V R N-A 1 b$ and $V R N-A 1 d$ with mutations in the promoter region (Figure 2 and 3; Table 2). These alleles occur in different polyploid wheats both tetra- and hexaploid but they have not been found in diploid wheat species $[23,32]$. We did not find the dominant VRN-A1a allele with insertion of a foldback element in the promoter region amongst the $T$. dicoccoides accessions (Table 1 ). This allele is abundant in hexaploid wheat T. aestivum $[23,35,36]$.

PCR analysis of the $1^{\text {st }}$ intron region revealed allele $V R N-A 1 L$ in three accessions of T. dicoccoides (Figure 4 and 5). This allele is common among cultivated spring varieties of tetraploid T. durum [24,37]. VRN-A1L was not found in the diploid wheat species.

Unlike $V R N-A 1$, locus $V R N-B 1$ displayed no significant variability within either the promoter or $1^{\text {st }}$ intron regions (Figure 2, Additional file 2). Several large mutations, mainly deletions within the $1^{\text {st }}$ intron of VRN$B 1$ were previously found in spring varieties of $T$. aestivum $[24,25,27,35]$. But these mutations have not yet been detected in tetraploid wheat species, and are thus likely to have a later origin.

The analysis of $V R N-1$ polymorphism in polyploid and diploid wheat accessions showed that the two groups differ with respect to the sets of $V R N-1$ alleles found among accessions.

\section{Effect of VRN-1 gene structure on growth habit Diploid wheat species}

We determined the impact of $V R N-1$ alleles on growth habit in a selected set of accessions representing three diploid species of the A genome (Table 2). Out of five tested accessions of T. monococcum, four were spring 
types despite the presence of an intact promoter region at VRN-A1. In two of them spring growth habit was associated with insertion in intron 1 (VRN-A1ins). Totally, we found the VRN-A1ins allele in $31 \%$ of T. monococcum accessions (Additional file 1).

Previous data support the importance of intron 1 in the vernalization response and heading time determination, and the proposed key regulatory region has been narrowed to a highly conserved $2.8 \mathrm{~kb}$ "vernalization critical region" located near the left side of intron 1 [24]. A number of mutations (deletions, insertions etc.) in this region have been associated with high levels of $V R N-1$ transcripts and a spring growth habit in different crops $[24,26,31,38,39]$. It is suggested that these mutations may affect epigenetic chromatin states, resulting in a higher basal level of $V R N-1$ expression [19,26,40,41].

Dubcovsky et al. suggested that mutation in CArG- box of the VRN-A1f allele of T. monococcum results in spring habit preventing interaction with unknown repressor dependent on photoperiod [31]. However, we can not discriminate the effect of this allele on vernalization response, because in our material it always occurs in combination with VRN-Alins (Table 2; Additional file 1).

In two accessions of $T$. monococcum (TRI 17025, 17973) and one accession of T. boeoticum (TRI 17072) spring growth habit was associated with the wild recessive $V R N-A 1$ allele (VRN-A1; no insertion in intron 1; Table 2). Alignment of $V R N-1$ sequence from accessions TRI 17072 and TRI 17062 of T. boeoticum (spring and winter types, respectively) showed that the first accession had a mutation that has not been described previously, the substitution $\mathrm{T} \rightarrow \mathrm{C}$ coupled with 1 bp deletion about 200 bp downstream from the start of intron 1. It remains unclear whether this minor alteration within "critical region" (see above) is the reason for spring growth habit.

Previous studies of vernalisation genes in wheat have identified loss-of function mutations at the $V R N-2$ gene, the flowering repressor, which result in up-regulation of $V R N-1$ regardless of its allelic status. [15,17,31]. We studied 3 above mentioned accessions using previously developed molecular markers for $V R N-2$ gene [17] and found that accession TRI 17025 of T. monococcum contains a mutated recessive $V R N-2$ allele (data not presented). We failed to define the reason for spring growth habit in accession TRI 17973 of T. monococcum. In previous study, $6 \%$ of accessions of this species were of spring type despite the presence of $v r n-1 / V R N-2$ genotype [17]. Based on these data, it was suggested that genes other than $V R N-1$ and $V R N-2$ influence vernalisation response in diploid wheat.

Out of four accessions of T. boeoticum containing VRN$A 1 h$ allele and studied for vernalization requirements, two were of spring type, while the other two were associated with the winter habit (Table 2).
One plausible hypothesis has been that the spring growth habit of T. monococcum originated in wild populations of $T$. boeoticum and was later selected by man through cultivation [32]. However, absence of the VRN$1 \mathrm{~h}$ allele in $T$. monососсиm coupled with the presence of specific alleles conferring spring growth habit (VRN-A1f, $V R N-A 1 i n s)$ in this diploid leads us to propose an alternative hypothesis, of selection of independent mutations that appeared in T. monococcum during domestication.

We found no spring forms in T. urartu (Table 2). None of the $T$. urartu accessions included in this study had changes in the promoter or $1^{\text {st }}$ intron regions of $V R N-1$ which might confer a spring growth habit (Additional file 1). According to Dorofeev et al. [6], all forms of T. urartu are winter forms. Goncharov [42] found only $2 \%$ of $T$. urartu accessions to be spring types. In another previous study, no differences in the promoter region of $V R N-1$ relative to the recessive allele were found in four spring accessions of $T$. urartu [32].

\section{Tetraploid wheat}

In $T$. dicoccoides we found several $V R N-1$ alleles which are widely distributed among cultivated wheat polyploids. Allele $V R N-A 1 b$ is not always associated with spring growth habit in hexaploid wheat lines [43], in our study this allele was associated with winter habit in three accessions of $T$. dicoccoides (Table 2). Allele VRN-A1d influences growth habit more strongly, probably due to an additional deletion affecting the CArG box (Figures 2 and 3). It confers spring growth habit in all accessions of $T$. dicoccoides both studied here and from databases. Allele VRN-A1L previously found in many cultivated tetraploid forms of wheat [37] is also associated with dominant spring type (Table 2).

The question of appearance of spring growth habit in T.diccocoides remains open. This habit could be inherited from ancestral diploids, in particular, from ancestral form of T.urartu, precursor of A genome. However, as shown above, $T$. urartu is predominantly winter species. Kato et al. [44] studied geographical variation in the vernalisation requirements of $T$. dicoccoides and found that the distribution of spring forms was sporadic and restricted to warmer areas. The authors suggest that the spring type might have evolved from a previous winter prototype of $T$. dicoccoides as an adaptation to warmer conditions. Our data are consistent with this hypothesis implying that the VRN-1 associated determinants of spring growth habit in polyploid wheat were formed after combining of diploid genomes.

\section{Conclusions}

In the present study we investigated variability in the promoter and $1^{\text {st }}$ intron regions of the vernalization gene $V R N-1$ in different accessions of wild tetraploid species 
T. dicoccoides and its diploid A genome progenitors. Our results indicated that a number of $V R N-1$ alleles characteristic of polyploid wheats (VRN-A1b,VRN-A1d, $V R N-A 1 L)$ are found in $T$. dicoccoides, while others (VRN-A1a, VRN-B1 alleles) probably arose during later stages of polyploid evolution and were selected during domestication. The occurrence of spring forms in the diploid A genome progenitor species is only partially attributed to $V R N-1$ variation, since some of these forms contained an intact recessive $V R N-1$ allele, while others displayed a winter habit despite the presence of a modified $V R N-1$ allele $(V R N-A 1 h)$. The most abundant allele conferring spring growth habit in diploids was VRNAlins of T. monococcum, containing a $0.5 \mathrm{~kb}$ insertion in the $1^{\text {st }}$ intron. A higher variability of $V R N-1$ loci in polyploid species in comparison with diploids may be explained by the hypothesis that stresses due to allopolyploidization may provoke genome instability [45]. Greater $V R N-1$ loci variability gives polyploid wheat an advantage in conferring adaptation to a broader range of environments.

\section{Methods}

\section{Plant material and DNA extraction}

Plant material included 80 accessions of the wild tetraploid wheat species $T$. dicoccoides (BBAA) and the diploid A genome species T. monococcum, T. boeoticum and T. urartu (61, 59 and 40 accessions, respectively). These accessions were selected from collections in the Leibniz Institute of Plant Genetics and Crop Plant Research (IPK, Gatersleben, Germany), the ICARDA genbank (Syria) and the Vavilov All-Russian Institute of Plant Industry RAN (St Petersburg, Russia). Accession numbers, phenotypes and genotypes are given in Additional file 1.

Total genomic DNA was extracted from etiolated seedlings as described by Plaschke et al. [46] with modifications. Leaves (from 5 seeds per accession) were pooled, placed in racked collection tubes (24 per rack) and homogenised directly in extraction buffer using a FastPrep-24 (MP Biomedicals, USA).

\section{PCR}

PCR primers reported in Yan et al. [23], Fu et al. [24], Shcherban et al. [25] were used to detect the presence of dominant or recessive alleles of $V R N-A 1$ and $V R N-B 1$ loci in diploid and polyploid wheats (Table 1). To further discriminate different alleles in promoter regions of both loci we digested corresponding PCR products with restriction endonuclease $M s p$ I followed by separation of DNA fragments in 2-3\% high resolution agarose gel (HydraGene Co., China). The polymorphic 1-bp deletion (VRN-A1f) was detected as described by Dubcovsky et al. [31]. Using primers VRN1F_F (5'-ACAGCGGCTATGCTCCAGAC-3') and VRN1F_R (5'-GGAGGATGGCCAGGCCAAATC-3'), the second nucleotide of the reverse primer (underlined $\mathrm{T}$ ) was mutated to generate a Taq I restriction site for the $v r n-A 1$ allele that was absent in the VRN-A1f allele.

The sequences of the $T$. monococcum VRN-A1 gene (AY188331, DQ146421, DQ146422, DQ146423) and respective sequences of T.aestivum (AY747600, AY747601) were used to design specific primers flanking an insertion of $\sim 0.5 \mathrm{~kb}$ and deletion of $1.4 \mathrm{~kb}$ in the $1^{\text {st }}$ intron of $V R N-$ A1 (Table 1; Figure 4).

PCR was performed using a DNA Thermal Cycler 480 (Perkin Elmer Cetus, USA). Reaction mixtures were in a volume of $20 \mu \mathrm{l}$ containing 50-100 ng of genomic template DNA, $1 \mathrm{ng}$ of each of primer, $0.25 \mathrm{mM}$ of each dNTP, $1 \mathrm{x}$ reaction buffer $(67 \mathrm{mM}$ TrisHCl, $\mathrm{pH} 8.8$; $2 \mathrm{mM} \mathrm{MgCl} ; 18 \mathrm{mM}\left(\mathrm{NH}_{4}\right)_{2} \mathrm{SO}_{4} ; 0.01 \%$ Tween 20$)$ and 1 unit Taq polymerase. After initial denaturation at $94^{\circ} \mathrm{C}$ for $2 \mathrm{~min}, 35$ cycles were run at $94^{\circ} \mathrm{C}$ for $1 \mathrm{~min}, 55-61^{\circ} \mathrm{C}$ (depending on the primer pair used) for $1 \mathrm{~min}$, and $72^{\circ} \mathrm{C}$ for 0.5-2 min, followed by a final extension at $72^{\circ} \mathrm{C}$ for $5 \mathrm{~min}$. PCR products were separated on $1 \%$ agarose gel, stained with ethidium bromide and visualized under UV light.

\section{Sequencing of PCR products}

Amplified DNA fragments were excised following electrophoresis, purified using a QIAquick PCR purification kit (QIAGEN, Germany) and directly sequenced using an ABI PRISM Dye Terminator Cycle Sequencing ready reaction kit (Perkin Elmer Cetus, USA) and corresponding specific primers. Sequencing was performed in an ABI PRISM 310 Genetic Analyzer (Perkin Elmer Cetus). All obtained sequences were deposited to GenBank (Table 2).

\section{Evaluation of growth habit}

Growth habits of the diploid and tetraploid accessions containing different VRN-A1 alleles were determined from two replicates in 2013-2014 (Table 2). Seeds were sown in the greenhouse (ICG, Novosibirsk) without vernalization under a long day photoperiod regime. Three months after sowing, when all spring standard varieties had headed, experimental materials were classified as either spring (ear emergence) or winter (no visible ear formation) types.

\section{Additional files}

Additional file 1: Polymorphisms at the promoter and $1^{\text {st }}$ intron regions of $V R N-A 1$ gene in $\mathrm{X}$ accessions of diploid Triticum species and $Y$ accessions of tetraploid $T$. dicoccoides.

Additional file 2: Variability at VRN-B1 locus in T. dicoccoides. a- PCR amplification using specific primers P2/P5 to detect variation within the $V R N-B 1$ promoter region in different accessions of T. dicoccoides. b- Msp I restriction digestion of corresponding PCR products. C- PCR amplification with primers Intr1/B/F//Intr1/B/R4 to detect the absence of deletions in the $1^{\text {st }}$ intron of $V R N-B 1$. Asterisks mark the accessions for which the VRN-B1 promoter region was sequenced (Genbank: KM586661-65). 


\section{Competing interests}

The authors declare that they have no competing interests.

\section{Authors' contributions}

AS designed the study, carried out the molecular and bioinformatics experiments and greenhouse analyses, and prepared the manuscript. KS contributed to the molecular analysis. EAS helped with interpretation of the results and critically revised the manuscript. All authors read and approved the final manuscript.

\section{Acknowledgements}

We are grateful to Dr. A.Böerner and Dr. A.Graner (Leibniz Institute of Plant Genetics and Crop Plant Research, Gatersleben, Germany) for supplying seeds of wheat species. The study of diploid species was done in framework of the State Budget Programme (Project No VI.53.1.5.), the analysis of T. dicoccoides was supported by the Russian Scientific Foundation (Project No. 14-14-00161).

\section{Received: 5 December 2014 Accepted: 16 March 2015} Published online: 31 March 2015

\section{References}

1. Ozkan H, Willcox G, Graner A, Salamini F, Kilian B. Geographic distribution and domestication of wild emmer wheat (Triticum dicoccoides). Genet Resour Crop Evol. 2011;58:11-53.

2. Dvorak J, Zhang HB. Variation in repeated nucleotide sequences sheds light on the phylogeny of the wheat B and $G$ genomes. Proc Natl Acad Sci U S A. 1990;87:9640-4.

3. Kilian B, Ozkan H, Deusch O, Effgen S, Brandolini A, Kohl J, et al. Independent wheat B and $\mathrm{G}$ genome origins in outcrossing Aegilops progenitor haplotypes. Mol Biol Evol. 2007;24:217-27.

4. Salina EA, Lim YK, Badaeva ED, Shcherban AB, Adonina IG, Amosova AV, et al. Philogenetic reconstruction of Aegilops section Sitopsis and the evolution of tandem repeats in the diploids and derived wheat poliploids. Genome. 2006:49:1023-35.

5. Johnson BL, Dhaliwal HS. Reproductive isolation of T. boeoticum and T. urartu and the origin of the tetraploid wheats. Am J Bot. 1976;63:1088-96.

6. Dorofeev VF, Filatenko AA, Migushova EF, Udaczin RA, et al. Wheat. In: Flora of cultivated plants. Volume 1. Edited by Dorofeev VF, Korovina ON. Leningrad (St. Petersburg): Kolos (In Russian); 1979, 346 pp.

7. Konovalov FA, Goncharov NP, Goryunova S, Shaturova A, Proshlyakova T, Kudryavtsev A. Molecular markers based on LTR retrotransposons BARE-1 and Jeli uncover different strata of evolutionary relationships in diploid wheats. Mol Genet Genomics. 2010;283:551-63.

8. Dvorak J, McGuire PE, Cassidy B. Apparent sources of the A genomes of wheats inferred from polymorphism in abundance and restriction fragment length of repeated nucleotide sequences. Genome. 1988;30:680-9.

9. Dvorak J, Di Terlizzi P, Zhang HB, Resta P. The evolution of polyploid wheats: identification of the A genome donor species. Genome. 1993:36:21-31.

10. Friebe B, Kim NS, Kuspira J, Gill BS. Genetic and intercytogenetic analyses of the A genome of Triticum monococcum. VI. Production and identification of primary trisomics using the C-banding technique. Genome. 1990;33:542-55.

11. Hammer K, Filatenko AA, Korzun V. Microsatellite markers - a new tool for distinguishing diploid wheat species. Genet Resour Crop Evol. 2000;47:497-505.

12. Baum BR, Bailey LG. The origin of the A genome donor of wheats (Triticum: Poaceae) - a perspective based on the sequence variation of the 5S DNA gene units. Genet Resour Crop Evol. 2004;51:183-96.

13. Heun M, Haldorsen S, Vollan K. Reassessing domestication events in the Near East: einkorn and Triticum urartu. Genome. 2008;51:444-51.

14. Kilian B, Ozkan H, Walther A, Kohl J, Dagan T, Salamini F, et al. Molecular diversity at 18 loci in 321 wild and 92 domesticate lines reveal no reduction of nucleotide diversity during Triticum monococcum (einkorn) domestication: implications for the origin of griculture. Mol Biol Evol. 2007:24:2657-68

15. Distelfeld A, Li C, Dubcovsky J. Regulation of flowering in temperate cereals. Curr Opin Plant Biol. 2009:12:1-7.

16. Yan L, Loukoianov A, Tranquilli G, Helguera M, Fahima T, Dubcovsky J. Positional cloning of the wheat vernalization gene VRN1. Proc Natl Acad Sci U S A. 2003;100:6263-8.
17. Yan L, Loukoianov A, Blechl A, Tranquilli G, Ramakrishna W, SanMiguel P. The wheat VRN2 gene is a flowering repressor down-regulated by vernalization. Science. 2004;303:1640-4.

18. Yan L, Fu D, Li C, Blechl A, Tranquilli G, Bonafede M, et al. The wheat and barley vernalization gene VRN3 is an orthologue of FT. Proc Natl Acad Sci U S A. 2006;103:19581-6.

19. Trevaskis B, Hemming MN, Dennis ES, Peacock WJ. The molecular basis of vernalization induced flowering in cereals. Trends Plant Sci. 2007;12:352-7.

20. Murai K, Miyamae M, Kato H, Takumi S, Ogihara Y. WAP1, a wheat APETALA1 homolog, plays a central role in the phase transition from vegetative to reproductive growth. Plant Cell Physiol. 2003;44:1255-65.

21. Shitsukawa N, Ikari C, Shimada S, Kitagawa S, Sakamoto K, Saito H, et al. The einkorn wheat (Triticum monococcum) mutant, maintained vegetative phase, is caused by a deletion in the VRN-1 gene. Genes Genet Syst. 2007:82:167-70.

22. Trevaskis B, Hemming MN, Peacock WJ, Dennis ES. HVVRN2 responds to day-length, whereas HVVRN1 is regulated by vernalization and developmental status. Plant Physiol. 2006;140:1397-405.

23. Yan L, Helguera M, Kato K, Fukuyama S, Sherman J, Dubcovsky J. Allelic variation at the VRN-1 promoter in polyploid wheat. Theor Appl Genet. 2004;109:1677-86.

24. Fu D, Szucs P, Yan L, Helguera M, Skinner JS, vonZitzewitz J, et al. Large deletions within the first intron in VRN-1 are associated with spring growth habit in barley and wheat. Mol Gen Genomics. 2005;273:54-65.

25. Shcherban AB, Efremova TT, Salina EA. Identification of a new Vrn-B1 allele using two near-isogenic wheat lines with difference in heading time. Mol Breeding. 2012;29(3):675-85.

26. Shcherban AB, Khlestkina EK, Efremova TT, Salina EA. The effect of two differentially expressed wheat VRN-B1 alleles on the heading time is associated with structural variation in the first intron. Genetica. 2013;141:133-41

27. Milec Z, Tomkova L, Sumikova T, Pankova K. A new multiplex PCR test for the determination of $V r n-B 1$ alleles in bread wheat (Triticum aestivum L.). Mol Breeding. 2012;30(1):317-23.

28. Goncharov NP. Genetic resources of wheat related species:The Vrn genes controlling growth habit (spring vs. winter). Euphytica. 1998;100:371-6.

29. Stelmakh AF. Genetic effects of Vrn genes on heading date and agronomic traits in bread wheat. Euphytica. 1993;65:53-60.

30. Iwaki K, Haruna S, Niwa T, Kato K. Adaptation and ecological differentiation in wheat with special reference to geographical variation of growth habit and Vrn genotypes. Plant Breed. 2001;120:107-14.

31. Dubcovsky J, Loukoianov A, Fu D, Valarik M, Sanchez A, Yan L. Effect of photoperiod on the regulation of wheat vernalization genes $\mathrm{V} r n-1$ and Vrn-2. Plant Mol Biol. 2006;60:469-80.

32. Golovnina KA, Kondratenko E, Blinov AG, Goncharov NP. Molecular characterization of vernalization loci VRN1 in wild and cultivated wheats. BMC Plant Biol. 2010;10:168.

33. Middleton CP, Senerchia N, Stein N, Akhunov ED, Wicker T, Kilian B. Sequencing of chloroplast genomes from wheat, barley, rye and their relatives provides a detailed insight into the evolution of the Triticeae tribe. PLoS. 2014;9(3):e85761. doi:10.1371/journal.pone.0085761.

34. Dvorak J, Akhunov E. Tempos of gene locus deletions and duplications and their relationship to recombination rate during diploid and polyploid evolution in the Aegilops-Triticum alliance. Genetics. 2005;171:323-32.

35. Santra DK, Santra M, Allan RE, Campbell KG, Kidwell KK. Genetic and molecular characterization of vernalization genes $V r n-A 1, V r n-B 1$ and $V r n-D 1$ in spring wheat germplasm from the Pacific Northwest Region of the USA. Plant Breeding. 2009;128:576-84.

36. Shcherban AB, Emtseva MV, Efremova TT. Molecular genetical characterization of vernalization genes $V r n-A 1, V_{r n}-B 1$ and $V r n-D 1$ in spring wheat germplasm from Russia and adjacent regions. Cereal Res Commun. 2012:40(3):425-35

37. Oliveira HR, Campana MG, Jones H, Hunt HV, Leigh F, Redhouse DI, et al. Tetraploid Wheat Landraces in the Mediterranean Basin: Taxonomy, Evolution and Genetic Diversity. PLoS One. 2012;7(5):e37063.

38. von Zitzewitz J, Szucs P, Dubcovsky J, Yan L, Francia E, Pecchioni N, et al. Molecular and structural characterization of barley vernalization genes. Plant Mol Biol. 2005:59:449-67.

39. Hemming MN, Peacock WJ, Dennis ES, Trevaskis B. Low-temperature and day-length cues are integrated to regulate FLOWERING LOCUS T in barley. Plant Physiol. 2008;147:355-66. 
40. Oliver SN, Finnegan EJ, Dennis ES, Peacock WJ, Trevaskis B. Vernalization-induced flowering in cereals is associated with changes in histone methylation at the VERNALIZATION1 gene. Proc Natl Acad Sci U S A. 2009;106:8386-91.

41. Oliver SN, Deng W, Casao MC, Trevaskis B. Low temperatures induce rapid changes in chromatin state and transcript levels of the cereal VERNALIZATION1 gene. J Exp Bot. 2013;64:2413-22.

42. Goncharov NP. Comparative genetics of wheats and their related species. Novosibirsk: Siberian University Press (In Russian); 2002. p. 251.

43. Pidal B, Yan L, Fu D, Zhang F, Tranquilli G, Dubcovsky J. The CArG-box located upstream from the transcription start of wheat vernalization gene $V R N-1$ is not necessary for the vernalization response. J Heredity. 2009;100:355-64.

44. Kato K, Mori Y, Beiles A, Nevo E. Geographical variation in heading traits in wild emmer wheat, Triticum dicoccoides. I. Variation in vernalization response and ecological differentiation. Theor Appl Genet. 1997;95:546-52.

45. Levy AA, Feldman M. The impact of polyploidy on grass genome evolution. Plant Physiol. 2002;130:1587-93.

46. Plaschke J, Ganal MW, Roeder MS. Detection of genetic diversity in closely related bread wheat using microsatellite markers. Theor Appl Genet. 1995;91:1001-7.

\section{Submit your next manuscript to BioMed Central and take full advantage of:}

- Convenient online submission

- Thorough peer review

- No space constraints or color figure charges

- Immediate publication on acceptance

- Inclusion in PubMed, CAS, Scopus and Google Scholar

- Research which is freely available for redistribution 